\title{
Intelligent Walking Stick for Elderly and Blind People
}

\author{
Shalini Singh \\ ACSD, C-DAC \\ Mohali, India
}

\author{
Dr. Balwinder Singh \\ ACSD, C-DAC \\ Mohali, India
}

\begin{abstract}
Mobility for blind and elderly people is a problem as they have to face many difficulties in their day to day life. Most commonly, stick is use by blind and elderly people as a support for their body to stand and walk. The most common disadvantage of walking stick is user should have to be in close proximity of obstacle so that he can sense the location of obstacle to avoid bumping while waggling. We proposed a new concept of walking stick which is helpful for both blinds and elderly people to move in unfamiliar environment and monitor their medical condition. In this methodology we have used different sensors mounted on a walking stick which will help a user to navigate easily indoor and outdoor along with monitoring their health. Unlike other electronic blind stick which were helpful in detecting obstacle and alert user we designed a stick having multiple feature embedded in a single stick. This stick used for detecting obstacle, pit, water on ground to some level, location of user, pulse rate detection, body temperature measurement of user and alert another person by sending message about the health issue of user or some other emergency.
\end{abstract}

Keywords-Walking stick, TCRT1000, DYPME-007, GSM, GPS, electrodes, infrared sensor, thermistor, health monitoring.

\section{INTRODUCTION}

According to the World Health Organization, there are 285 million people projected to be visually impaired around the globe. There are 50 million people estimated to be blind who are of prolific age and need independent mobility to work or walk outside and be part of the mainstream and do all other daily essential works. There are 12 million people in India with blindness, the greatest number for any country in the world, according to 2011 census data. The earlier used white cane has major issues as subject need to be in close proximity of obstacle and sense the location of the obstacle by the tip of the white can. While sensing obstacle by the white can he could get hit to other obstacles around him. White can gives no knowledge of the obstacle nearby. The designed smart walking stick is equipped with different sensors which will alert user about various obstacles coming ahead in his way while walking. This smart walking stick has health monitoring features. User can check their pulse rate and body temperature using those health monitoring features. GPS and GSM modules are equipped in stick to track location of user and send an alert message to other person. Another important feature of this walking stick is voice alert system. The user will be notified by voice alert about obstacle, pit, wet surface and health issues. This will ease mobility of blind person as he will get attentive after listening voice alerts. Other embedded important features of this smart stick are object detection, pit detection, water detection. These features of smart walking stick are helpful in guiding elderly and blind users in their mobility and to avoid accidents. With the help of this smart walking stick user can walk freely and easily in unknown environment without depending on other person.

\section{PROPOSED WORK}

The methodology was planned to develop a smart electronic stick having different features which were absent in a conventional stick. Technology enhancement over the conventional method is significant so that blind and elderly people make their lives better by providing them a feeling of safety, security and comfort as much as possible whenever and wherever they are. This methodology aims to help blind and elder users with monitoring their health and daily movement issues. For this we have designed a smart electronic walking stick which helps users to move independently and confidently. The circuit of smart stick consists of ultrasonic sensor which is used for detecting obstacle in front of user, PIR sensor is used to detect the pits on the roads and uneven surfaces, a pair of electrodes are fitted at bottom to check water on road, TCRT1000 sensor is used for detecting pulse rate of user, temperature sensor is used for detecting body temperature, GPS module is used to track the location of user and GSM module for sending message to the concerned person so he will be notified about the user condition. This stick is helpful for blinds and elderly people in terms of health also and they can continuously monitor their pulse rate and body temperature without the need of any other instrument whose maintenance is also a big problem for blind and elderly people. There is an alert switch on walking stick if user feels uneasy about their health or they want to alert other person regarding any problem any time, they can press panic switch and an alert message with the location of the user is send to the concerned person. The main purpose of this methodology is to help disabled people by advancing the techniques used earlier so that they will confidently and easily spend their life without any dependence. We have embedded the health monitoring features in smart stick because in blind and elder people health is major issue and this walking stick health monitoring features will be a comfort. This stick will give them a feeling of relief and liberation and they always feel connected to some person whom they wanted to associate in case of emergency by sending an alert whenever needed. To advance the earlier designed electronic walking stick we have adjoin all necessary solutions in a single walking stick to the problematical concerns of daily life of elderly and blind users. 


\section{METHEDOLOGY}

The designing of electronic walking stick for the blind, including a supporting rod having a handle structure mounted on the upper end of rod, handle structure containing a power switch, different sensors, control box and an audio alert system. Smart stick equipped with several sensors positioned at different height of stick according to requirement and this allows ease of operation. Smart stick is used to detect and notify different parameters so that user will avoid bumping.

This stick has several integrated subsystems. The stick is integrated with power supply, microcontroller, ultrasonic sensor, PIR sensor, electrodes, pulse rate sensor, temperature sensor and audio module along with GPS and GSM module. The system is incorporated with PIC16F877A microcontroller which operates the whole system. When smart stick initializes it will activate all attached sensors and start detecting obstacle nearby. If obstacle found ahead in close proximity of certain amount of distance (here $10 \mathrm{~cm}$ ), the ultrasonic sensor will alert user by audio alert that the obstacle is near and displays the obstacle distance in $\mathrm{cm}$ on LCD screen. There is a push switch at the bottom of stick and the PIR sensor is placed about $10 \mathrm{~cm}$ ahead of stick when the stick hit the ground the push switch gets pressed and it will activate the PIR sensor. The PIR sensor starts detecting if there is any pit at the bottom of PIR sensor. If there is any pit, it will notify user by audio alert that pit is detected and displays on LCD as well.

Electrodes are placed $3 \mathrm{~cm}$ above from the bottom end of the stick so that if there is water of large amount on surface electrodes will detect it and user will be alarm by audio alert and by displaying on LCD that water is detected. There is pulse sensor and temperature sensor placed near handle of stick so that when user want to check his pulse he can press the pulse switch and place his finger on pulse sensor the sensor will detect pulses and if the pulse count lies in normal range it will display pulse rate is normal otherwise it displays pulse rate is abnormal and alert the user by audio alert too. For measuring body temperature we have used temperature sensor when user place his finger on temperature sensor it will display measured temperature of user's body and display the measured temperature on LCD. If the measured temperature lies outside the normal range, it will generate an audio alert.

If the user is not feeling comfortable while walking or even while sitting, he/she can use the alert switch provided on the walking stick. This alert switch when pressed by the user sends an alert message to some pre-stored mobile number using GSM module that the user is not feeling well along with its location as latitude and longitude determined using GPS module. The block diagram of proposed methodology is shown in figure I.

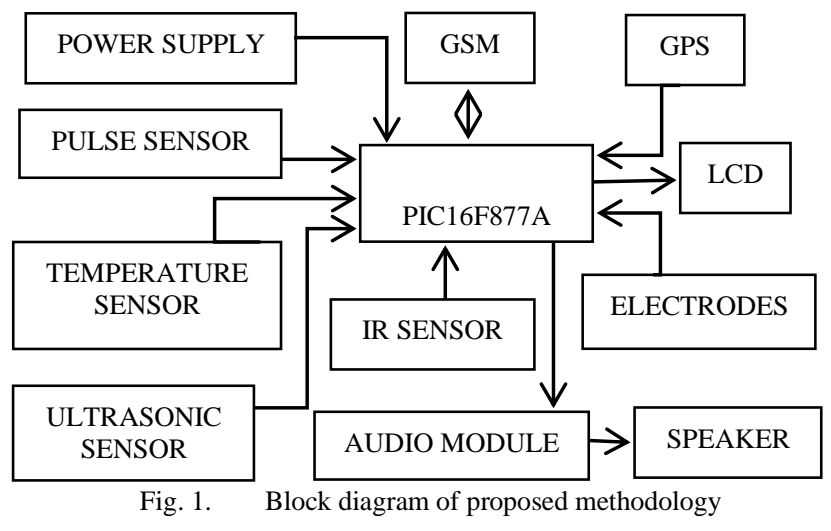

\section{RESULT AND DISCUSSION}

During the evaluation of the design and development of Smart Sensor Based Walking Stick it was tested in practicable and real time conditions. It is also evaluated by blind person. There are different conditions on different routes have been chosen to test the performance. In first route, we have placed dummy of wall as obstacles in numbers. The blind person was unfamiliar with this place. At first Blind person used the white cane on this route and comparative performance with white can has been evaluated. The route was $27 \mathrm{~m}$ long in distance and it was covered at a speed of $0.522 \mathrm{~m} / \mathrm{s}$ with help of white cane. But when blind person used smart stick it was covered at speed of $0.78 \mathrm{~m} / \mathrm{s}$ and without any problem. In the end route shows that "Smart walking stick" has outstanding performance as compared to white cane. Likewise first route we got excellent results when we tested this stick in other routes as well.

A comparison of different routes and the performance of this smart walking stick as compare to white can are shown in the table I below

TABLE I. Name of the Table that justify the values

\begin{tabular}{|c|c|c|c|}
\hline \multirow{2}{*}{ Path } & Length(m) & \multicolumn{2}{|c|}{ Performance of white stick } \\
\cline { 3 - 4 } & & $\begin{array}{c}\text { Time } \\
\text { taken(sec) }\end{array}$ & Speed(m/s) \\
\hline A & 30 & 64 & 0.47 \\
\hline B & 15 & 24 & 0.62 \\
\hline C & 27 & 52 & 0.52 \\
\hline D & 20 & 34 & 0.58 \\
\hline E & 25 & 40 & 0.62 \\
\hline
\end{tabular}

Table II show the performance of the Smart Walking Stick in terms of time taken in seconds and speed in $\mathrm{m} / \mathrm{s}$. In the end if we compare the outcomes in different routes in Table I and Table II, it shows that "Smart walking stick" has outstanding performance as compared to white cane.

TABLE II. PERFORMANCE OF SMART WALKING STICK

\begin{tabular}{|c|c|c|c|}
\hline \multirow{2}{*}{ Path } & \multirow{2}{*}{ Length(m) } & \multicolumn{2}{|c|}{ Performance of white stick } \\
\cline { 3 - 4 } & & Time taken(sec) & Speed(m/s) \\
\hline A & 30 & 42 & 0.71 \\
\hline B & 15 & 18 & 0.83 \\
\hline C & 27 & 35 & 0.77 \\
\hline D & 20 & 24 & 0.83 \\
\hline E & 25 & 28 & 0.89 \\
\hline
\end{tabular}




\section{A. Obstcle detection}

Obstacle detection is another feature that has been added in this smart stick. The obstacle detection circuit has provided an accuracy of approximately 4 feet. Data is collected from an ultrasonic sensor used to find the obstacle distance. The detection of obstacle is then shown on the 16X2 LCD. For a blind user who is unable to see the message on LCD, an audio message was delivered by APR33A3 module so that the user gets alert. In the Figure 3 below, an obstacle came in front of ultrasonic sensor which is about $3 \mathrm{~cm}$ far. The object gets detected by ultrasonic sensor and the calculated distance of obstacle from sensor is displayed on LCD which is shown in figure below. Figure 3 shows the usage of Smart Walking stick and Table III shows the various distance measurements and alerts given by the system.

TABLE III. DISTANCE MEASUREMENT (MINIMUM THRESHOLD DISTANCE 10CM)

\begin{tabular}{|c|c|c|}
\hline $\begin{array}{cc}\text { Actual } & \text { Obstacle } \\
\text { distance }(\mathrm{cm}) & \end{array}$ & \begin{tabular}{lr}
\multicolumn{2}{c}{ Measured } \\
distance & using \\
distance & measuring \\
unit & \\
$\quad(\mathrm{cm})$ &
\end{tabular} & $\begin{array}{l}\text { Voice Alert } \\
\text { by APR } \\
\text { (Yes/ No) }\end{array}$ \\
\hline 3 & 3 & Yes \\
\hline 5 & 5 & Yes \\
\hline 2 & 3 & Yes \\
\hline 6 & 5 & Yes \\
\hline 7 & 7 & Yes \\
\hline 10 & 10 & No \\
\hline 11 & 11 & No \\
\hline 13 & 13 & No \\
\hline 16 & 16 & No \\
\hline 17 & 17 & No \\
\hline 18 & 18 & No \\
\hline
\end{tabular}

\section{B. Pulse rate calculation}

Another feature that is added in this stick is the measurement of pulse rate. It is obvious that during old age the pulse rate of a person is not static because of the declining health conditions. High or low pulse rates may be an indication of some sort of illness. This problem is very much prominent in old age people especially when they walk. Hence we provided a pulse rate measurement system. We used TCRT1000 sensor for pulse sensing. In adults the normal resting pulse rate is considered in range of 60 to 100 beats per minute. So when the user holds the stick in his/her hand, his pulse rate gets calculated automatically. If the calculated pulse rate comes in range of 60 to 100, it will show pulse rate is normal otherwise it generates message on LCD

\section{Pit detection}

While walking there is a probability that a blind person can fall due to his stepping into small or even bigger pits. Hence there is a requirement to intimate him/her about any pit coming in his way whi9le he is walking. So we provided a passive infrared sensor, placed in the stick facing downward. Any hole or pit in way is detected by using this sensor. The signal from this sensor is fed into the microcontroller which decides whether there is a pit or not. On the detection of the pit, the user will be notified by voice alert and by displaying it on LCD. The range of detection can be set accordingly in this sensor. The detection range that we manually configured is the height from bottom of stick to sensor location so any pit below that fixed distance can be detected.

For testing this stick, it is carried by the user on the stairs. While walking on the stairs, the proximity sensor stops getting even surface and it detected uneven surface and shows pit detected. The user will be notified by displaying message on LCD and by voice alert which is generated by the APR system, that there is a pit and user will get attentive.

\section{Body temperature measurement}

The normal body temperate is considered $36.1 \pm 0.4{ }^{\circ} \mathrm{C}$. We have used NTC thermistor to detect the body temperature. By using the integrated temperature sensor, we get the user's temperature as $36^{\circ} \mathrm{C}$ which lies in normal range of human body temperature. The testing results are shown in Figure 6 . If the temperature is not normal i.e. if it is above $37^{\circ} \mathrm{C}$, the APR will give an audio alert.

\section{E. Water detection on road}

While walking, there may be water/mud on the surface of the road. If a person is blind or even if he/she has poor vision, then he can get into that muddy area spoiling his clothes or even getting hurt because of the slippery surface. For avoiding this, we have provided a system which alerts the user about the condition of the surface of road.

We have used electrodes in lower part of smart stick placed little above from the bottom to detect water on the surface. We dropped the designed stick in a glass filled with water. This level of water in the glass is kept comparable to the water/mud on road surface where it is difficult to walk. For testing we used a glass of water (indoor testing).

\section{F. Location nformation using GPS and GSM module}

When a blind or elderly person is out of home for walk or for other reason, if his/her health becomes suddenly poor i.e. if he feels that it is difficult for him to reach home back, he can intimate one of his relatives about this. There is an alert button provided on the stick, this button when pressed sends the coordinate of stick to a pre stored mobile number, i.e. "+91**********" via GSM module through SMS. The GPS module calculates the latitude and longitude of the location of the user.

\section{CONCLUSION}

The smart walking stick is more advance, easy to maintain, cheap, durable than conventional one. With the help of smart walking stick blind, elderly people can advance more than $15-20 \%$ travel speed, reduce minor collision; do not lose their way, and increase safety and confidence. Blind and elderly users can move confidently without any dependence on other person with the help of this smart stick. With the help of this walking stick user will feel secure and independent. This stick is very helpful in avoid accidents. Other person will remain in touch with the user via alerts. If there any problem occur, emergency alert regarding user health and his location will be send to related person and he will be notified immediately. Thus this is very helpful for both blind and 
elderly people both in terms of health monitoring and safe travelling. The future work can be the performance of stick can be improved by adding extra health monitoring features. We can add diabetes monitoring feature in stick as it is one of most arising problem in elderly persons. Future work will be focused on enhancing the performance of the system and reducing the load on the user by adding the camera to guide the blind exactly. Images acquired by using web camera and smart cameras helps in identification of objects as well as scan the entire instances for the presence of number of objects in the path of the blind determining long range target objects. The other scope may include a new concept of prime and safe path detection based on neural networks for a blind person.

It can also detect the material and shape of the object. The principles of mono pulse radar can be utilized for determining long range target objects. The other scope may include a new concept of prime and safe path detection based on neural networks for a blind person.

\section{ACKNOWLEDGEMENT}

The authors are thankful to faculty and staff of ACSD Department, C-DAC, for their support and guidance during the completion of this article

\section{REFERENCES}

[1] Namita Agarwal, Anosh Iyer, Sonalakshi Naidu, Snedden Rodrigues "Electronic Guidance System For The Visually Impaired - A Framework" 978-1-4799-8187-8/15 IEEE
[2] Shashank Chaurasia and K.V.N. Kavitha "An Electronic Walking Stick For Blinds" ISBN No.978-1-4799-3834-6 2014 IEEE

[3] Mohammad Hazzaz Mahmud, Rana Saha, Sayemul Islam “ Smart walking stick - an electronic approach to assist visually disabled persons" International Journal of Scientific \& Engineering Research, Volume 4, Issue 10, October-2013

[4] David T. Batarseh, Dr. Timothy N. Burcham, and Dr. Gary M. McFadyen "An Ultrasonic Ranging System for the Blind" 0-78033869-3197-1997IEEE

[5] G. Gayathri, M. Vishnupriya, R. Nandhini, Ms. M. Banupriya "Smart Walking Stick for Visually Impaired" IJECS Volume 3. Issue 3 March, 2014 Page No.4057-4061

[6] World Health Organization report on "Global data on visual impairments 2010" Available At http://www.who.int/blindness/GLOBALDATAFINALforweb.pdf

[7] David T. Batarseh, Dr. Timothy N. Burcham, and Dr. Gary M. McFadyen, M. Yusro, K.M. Hou, E. Pissaloux Shi, H. L. Ramli, K. Sudiana, "Concept and design of a smart environment explorer stick", in Proc. 6th international Conference on Human System lnteraction,vol.4,no.6, pp.70-77, June 2013.

[8] Saez, Juan Manuel, Francisco Escolano, and Miguel Angel Lozano. "Aerial ObstacleDetection With 3-D Mobile Devices", IEEE Journal of Biomedical and Health Informatics,2015.

[9] Sudeep Gupta, Ilika Sharma, Aishwarya Tiwariand and Gaurav Chitranshi "Advanced Guide Cane for the Visually ImpairedPeople"in Proc. 1st International Conference on Next Generation Computing Technologies, India, 4-5 September 2015

[10] N. Mahmud Saha, R.K. Zafar, R.B. Bhuian, M. B. H. Sarwar, "Vibration and voice operated navigation system for visually impaired person", International Conference on Informatics Electronics \& Vision, vol.6, no.1, pp.1-5, May 2014 